produce a repressive chromatin environment around the BDNF promoter.

The authors deduce that 2DG has anticonvulsant and antiepileptic properties, and believe this drug could become important in the treatment of epilepsy, founding a new class of antiepileptic drugs that target energy metabolism. They add that the findings relating to NRSF-mediated repression and its regulation by glycolysis have implications for the treatment of other diseases, including other neurological disorders and cancer.

Original article Garriga-Canut M et al. (2006) 2-DeoxyD-glucose reduces epilepsy progression by NRSF-CtBPdependent metabolic regulation of chromatin structure. Nat Neurosci 9: 1382-1387

\section{Symptoms of undiagnosed stroke are commonplace in the general population}

Undiagnosed strokes are common-using MRI, the 1998 ARIC study documented clinically silent cerebral infarctions in $11 \%$ of adults aged 55-64 years. The REGARDS study, published recently in the Archives of Internal Medicine, set out to further investigate the prevalence of stroke symptoms in a general US population (i.e. people who had not been diagnosed with stroke or a transient ischemic attack) and the association of these symptoms with recognized risk factors, as defined by the Framingham stroke risk score. Information was first obtained by telephone interview, with a physical examination performed 3-4 weeks later. A final cohort of 18,462 participants (mean age 65.8 years) was available for analysis.

One or more symptoms of stroke, such as sudden weakness, numbness or loss of vision, were reported by 3,292 subjects- $17.8 \%$ of the group. Multivariate analysis showed that stroke symptoms were more common among African American participants than white participants, and among participants with less education, lower income and poorer self-reported general health. There was a greater prevalence of stroke symptoms among subjects with high Framingham stroke risk scores. Targeted education and attempts to ameliorate risk factors such as hypertension, atrial fibrillation and smoking should be employed as preventative measures for patients who report symptoms of stroke. Follow-up of the REGARDS cohort will reveal whether or not those who reported symptoms and sought medical attention had actually experienced a stroke.

Original article Howard VJ et al. (2006) High prevalence of stroke symptoms among persons without a diagnosis of stroke or transient ischemic attack in a general population: the Reasons for Geographic And Racial Differences in Stroke (REGARDS) study. Arch Intern Med 166: 1952-1958

\section{Paclitaxel is a suitable chemotherapy for patients with metastatic brain tumors}

In metastatic brain tumors (MBT), P-glycoprotein (Pgp) is abundantly expressed on the surface of cerebral capillaries and is responsible for the multidrug resistance phenomenon. In human cancer cell lines, tamoxifen, a nontoxic inhibitor of Pgp, was shown to inhibit the multidrug resistance phenotype. The objective of the study by Fine et al. was to assess differences in the deposition of paclitaxel between MBT and primary brain tumors (PBT) and to examine whether tamoxifen influenced the deposition of paclitaxel.

The trial included 27 patients with PBT or MBT who received intravenous paclitaxel $\left(175 \mathrm{mg} / \mathrm{m}^{2}\right.$ over $\left.3 \mathrm{~h}\right)$ either alone or with tamoxifen $\left(160 \mathrm{mg} / \mathrm{m}^{2}\right.$ orally on days $1-5$ preceding paclitaxel). The investigators showed that there was no significant difference between the two study groups regarding paclitaxel concentration in the tumor center, periphery or surrounding normal tissue. MBT showed greater average paclitaxel concentrations in the center (1.93-fold, $P=0.10)$ and periphery of the tumor (2.46-fold, $P=0.039)$ compared with PBT, which correlated with earlier studies showing decreased Pgp expression in MBT.

Although further studies using higher and longer dosages of tamoxifen should be performed, this work is the first to show increased deposition of paclitaxel in MBT versus PBT. The authors suggest that paclitaxel may be more suitable as a chemotherapeutic agent for MBT, dependent upon the tumor's origin and the clinical effectiveness of paclitaxel for the primary tumor's histopathology. Conceptually, this may lead to an expanded number of available natural product chemotherapy drugs for the treatment of MBT.

Original article Fine RL et al. (2006) Randomized study of paclitaxel and tamoxifen deposition into human brain tumors: implications for the treatment of metastatic brain tumors. Clin Cancer Res 12: 5770-5776 